\title{
THE COMPACT NONTHERMAL RADIO SOURCE AT THE GALACTIC CENTER: AN UPDATE
}

\author{
K. Y. Lo \\ Astronomy Department, University of Illinois \\ $1011 \mathrm{~W}$. Springfield Avenue \\ Urbana, IL 61801 , U. S. A.
}

\begin{abstract}
We review the current observational status of Sgr A*, the compact nonthermal radio source at the galactic center. Sgr A* is a unique radio source at a unique location of the Galaxy. It is unlike any compact radio source associated with known stellar objects, but it is similar to extragalactic nuclear compact radio sources. The positional offset between Sgr A* and IRS16 places little constraint on the nature of the underlying energy source, since IRS16 need not be the core of the central star cluster. Sgr A* is still the best candidate for marking the location of a massive collapsed object.
\end{abstract}

\section{INTRODUCTION}

At the center of $\mathrm{Sgr} A$, the powerful radio source at the center of the Galaxy, is an extremely compact nonthermal radio source, by now commonly referred to as $\mathrm{Sgr} A * .1,2$ Such a radio source was anticipated as a possible signature of a massive black-hole that was proposed to be the energy source for the luminosities observed towards the center. ${ }^{3}$ Attempts to detect a compact source in Sgr A was first made by Ekers and Lynden-Bel1, ${ }^{4}$ and Downes and Martin, ${ }^{5}$ but their efforts were limited by the insufficient angular resolution of their interferometers. The compact nonthermal radio source was first unambiguously detected by Balick and Brown. ${ }^{1}$

Observations of Sgr A* and the constraints one can place on the nature of the underlying energy source have been recently reviewed in some detail. ${ }^{6}$ Here, we shall only discuss the new observational material that have been gathered since then, and discuss the implications and constraints that have been placed on Sgr A*.

\section{THE RADIO PROPERTIES OF SGR A*}

Efforts to determine the structure of Sgr A* have gone on for many years, 7-10 but because of the practical difficulties there is still no brightness distribution map of the source. The principal limitation, given the existing radio telescopes and the angular scale of the source, is that there are very few sufficiently short baselines on which the source 
is not completely resolved. Despite the lack of a good map, the properties of the radio source are reasonably well known, and they can be summarized in Table 1.

TABLE 1

Observed Properties of Sgr A*

$\begin{array}{ll}\text { Distance to the center } & 8.5 \mathrm{kpc} \\ \text { Source size } & <13 \mathrm{AU}\left(-1.7 \times 10^{14} \mathrm{~cm}\right) \\ \lambda \text {-dependence of size } & \lambda^{2 \pm 0.1}(\lambda \geq 1.35 \mathrm{~cm}) \\ \text { Axial ratio } & 0.55 \pm 0.25(\lambda=3.6 \mathrm{~cm}) \\ \text { Position angle of elongation } & 98^{\circ} \pm 15^{\circ}(\lambda=3.6 \mathrm{~cm}) \\ \text { Source expansion velocity } & <13 \mathrm{~km} / \mathrm{s} \\ \text { Flux density variability } & -0.2 \mathrm{C}(1974-1988) \\ \text { Spectral index } & \geq 0.25 \\ \text { Turnover frequency } & \geq 89 \mathrm{GHz}^{*} \\ \text { Radio luminosity } & -10^{34} \mathrm{erg} \mathrm{s}^{-1 *} \\ \text { Brightness temperature } & >7 \times 10^{8} \mathrm{~K}(\lambda=1.35 \mathrm{~cm}) \\ \text { Circular polarization } & <0.58\end{array}$

*Modulation index $(\sigma / S)$, cf Zhou et al, this volume. ${ }^{11}$

*If the inferred $\lambda 1 \mathrm{~mm}$ flux of $\mathrm{Sgr} \mathrm{A}^{*}$ is adopted, ${ }^{2}$ the turnover frequency is $\geq 230 \mathrm{GHz}$, and the radio luminosity is 3 times larger.

\subsection{Variability and Interstellar Scattering}

Before the intrinsic properties of $\mathrm{Sgr} A *$ can be definitively inferred, it is necessary to assess the importance of scattering by interstellar electrons on the radiation from $\mathrm{Sgr} \mathrm{A}^{*}$. Davies et al. ${ }^{9}$ first suggested that the observed size of $\mathrm{Sgr} \mathrm{A}^{*}$ is broadened by interstellar scattering, in order to explain the $\lambda$-dependence of the observed size. If scattering is important, the measured size will be merely an upper limit to the intrinsic size of the source.

Besides angular broadening of the source, interstellar scattering can cause rapid diffractive scintillation of the intensity, ${ }^{13}$ somewhat analogous to the earth's atmosphere causing twinkling of stars. Large scale irregularities in the electron distribution can act as lenses, focussing or defocussing the radiation, resulting in the slow refractive scintillation of the intensity. ${ }^{14,15}$ Therefore, studying flux variability of Sgr A* can help to gauge the importance of interstellar scattering. Recently, Zhou et a1 ${ }^{11}$ collated almost all the available flux density measurements of $\mathrm{Sgr} \mathrm{A}^{*}$ at different wavelengths since it was identified in 1974. The observed flux density varied but never by more than -30 percent $(\Delta S / 2 S$.) The modulation index (the ratio of the standard deviation to the mean value of the flux densities) appears to be independent of wavelength. The collated data suggest that the time scale ranges from possibly hours to years. The time scale appears to increase 
with increasing wavelength, ranging up to 5 years at $20 \mathrm{~cm}$. Since the discovery of Sgr A* 14 years ago, no large outburst of the flux density has been seen.

The variability of $\mathrm{Sgr} A *$ can be accounted for as refractive scattering by the irregularities in the ionized medium surrounding the galactic center. The inferred time scale implies a transverse velocity of the scattering screen relative to the line of sight connecting the source and the observer exceeding $1000 \mathrm{~km} / \mathrm{s}$, somewhat larger than the typical velocities observed at the center. ${ }^{11}$ However, the collated variability data have limited and uneven sampling, and a better determination of the variability time scale requires further observations. Refractive scattering can also produce fluctuations in the source position, as well as elongation of the source shape. ${ }^{16}$ Proper motion measurements of Sgr A* at different wavelengths already have put stringent limits on the wanderings of the source position and therefore on refractive scattering of the radiation from $\mathrm{Sgr} A * .16-18$

The scattering angle of $\mathrm{Sgr} A *$ is among the highest observed, and the amount of electron density fluctuations implied is uncomfortably large, although the exact value depends on where the bulk of scattering occurs. ${ }^{13}$ (See also Blandford, this volume.) There are indications that sources within 100 pc of the center have large observed sizes implying a source of strong scattering near the center, but Cordes et al ${ }^{19}$ suggest that the observed scattering in $\mathrm{Sgr} \mathrm{A}^{*}$ is a realistic extrapolation from pulsar results which can be explained by a two-component model of general interstellar electrons.

While the $\lambda^{2}$-dependence of the observed source size can be explained by interstellar scattering, it also could be due to a $1 / r$ distribution of thermal electrons within the source. Given the conflicting indications of the flux variability and the lack of fluctuations in source position, the evidence for refractive scattering of radiation from Sgr A* is still not definitive.

\subsection{Source Spectrum}

Sgr A has been mapped recently at $\lambda 1.3 \mathrm{~mm}$ with a $15^{\prime \prime}$ beam. ${ }^{12}$ The contribution of the free-free emission from ionized gas was subtracted from the $1.3 \mathrm{~mm}$ map by using a wavelength-scaled $2 \mathrm{~cm}$ map of $\mathrm{Sgr} A$ West. A compact but extended source remains after the subtraction. A $\lambda 1.3 \mathrm{~mm}$ flux density of $\sim 2$ Jy has been derived for Sgr A*. If this flux is synchrotron emission from Sgr A* itself, the turnover frequency of the synchrotron source will be extremely high, suggesting an extremely compact radio source.

\section{UNDERLYING ENERGY SOURCE FOR SGR A*}

\subsection{Ordinary Stellar Sources}

Even if the brightness distribution of $\mathrm{Sgr} \mathrm{A}^{*}$ is as yet unknown and the importance of interstellar scattering unsettled, it is clear the radio source has a very small dimension. The $10^{14} \mathrm{~cm}$ scale poses a very strong 
constraint on the underlying energy source and requires an object with stellar dimensions. In the Galaxy, there are very compact radio sources associated with known stellar objects - pulsars, binary stellar radio sources, young supernovae or supernova remnants. However, empirical arguments alone can exclude all of these as possible energy source. Ordinary pulsars are ruled out because the spectral index, $\alpha$, of pulsar radiation generally lies in the range $-3<\alpha<-1$, whereas for Sgr $A^{*}, \alpha>0.2$. The most luminous pulsars have an average radio luminosity of about $10^{4}$ times less than $\mathrm{Sgr} \mathrm{A}^{*}$. Furthermore, pulsars are very common, whereas Sgr $A^{*}$ is unique in the Galaxy. Sgr A* cannot be a young supernova remnant, which expands rapidly, since its expansion velocity is constrained to be $<13 \mathrm{~km} / \mathrm{s}$ averaged over 8 years.

Sgr $A^{*}$ is unlikely to be a binary stellar radio source because such sources are characterized by frequent, large outbursts in which $\Delta S / 2 S$ could be 10 to 1000 , while their steady radio luminosity is typically $10^{5}$ times smaller than that of $\mathrm{Sgr} \mathrm{A}^{*}$. The ratio of their $\mathrm{X}$-ray to radio luminosities is least 30 times larger than that of Sgr A*.

\subsection{Power Supplied by a Rotating Neutron Star ?}

While one cannot explain Sgr A* in terms of known stellar radio sources, the radio luminosity of $\mathrm{Sgr} \mathrm{A}^{*}$ is only $\sim 10^{34} \mathrm{erg} \mathrm{s}^{-1}$ or $\sim 10 \mathrm{~L}_{\bullet}$. As a rotating neutron star can supply up to $10^{38} \mathrm{erg} \mathrm{s}^{-1}$ of power, it can in principle power Sgr A*. A model of pulsar-driven wind was proposed for the radio emission of $\mathrm{Sgr} A *{ }^{20}$ The radio source has to be pressure confined by the pulsar moving through a dense gas cloud at high velocity. Recently, there is a similar model based on a neutron star embedded in a dense gas cloud with density $\sim 10^{8} \mathrm{~cm}^{-3} .21$ In this case, the small scale size and high radiu luminosity are set by the small coherence length in the magnetically tangled turbulent high density gas immediately surrounding the pulsar. The lack of expansion of $\mathrm{Sgr} A *$ depends on the pulsar's high velocity relative to the gas.

Independent of the plausibility of the assumptions involved, both models have problems when confronted with observations. First, there is a cavity in the gas distribution within the central few parsecs of the Galaxy. ${ }^{20}$ More importantly, the transverse motion of Sgr A* is observed to be $<40 \mathrm{~km} / \mathrm{s}^{16}$ An important point often overlooked in models involving rotating neutron stars is why more sources like Sgr A* are not found in other parts of the Galaxy.

\section{3 Proper Motion of Sgr A*}

The mass of $\mathrm{Sgr} \mathrm{A}^{*}$ is a critical parameter needed to determine the nature of the energy source. An indirect way of constraining its mass is to measure its proper motion. If $\mathrm{Sgr} \mathrm{A}^{*}$ is a stellar mass object near the bottom of the potential well at the center, very likely it will possess a large velocity. Hence, a measurable transverse velocity would rule out the possibility that it is supermassive. Proper motion results show that the motion of the sun is observed relatively to Sgr A* but not relative to the extragalactic reference sources. ${ }^{18}$ The latest upper limit on the

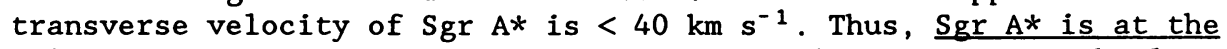
Galactic center, and not a background extragalactic source. The low upper 
limit to the transverse velocity, while not a proof, is consistent with Sgr A* being supermassive.

\section{SIMILARITY TO EXTRAGALACTIC COMPACT RADIO SOURCES}

While Sgr A* is a unique radio source at a unique location of the Galaxy, it has properties very similar to those of extragalactic compact radio sources. In particular, in the nucleus of $M 81$, a nearby bright spiral (Sb) galaxy, there exists such a radio source which has been quite well observed. It is compact (1000 AU $\times 4000 \mathrm{AU}$ ), with the major axis essentially aligned with the minor axis of the galaxy. ${ }^{23}$ It has a rising spectrum and is mildly variable. It is also a unique radio source at the center of a bright galaxy. ${ }^{24}$

Many galaxies harbor very powerful compact radio sources at their centers, with luminosities ranging up to $10^{46} \mathrm{erg} \mathrm{s}^{-1} \cdot{ }^{25} \mathrm{~A}$ unified model that can account for much of the nuclear acitvities ultimately depends on gas accreting onto a massive black-hole. ${ }^{26}$ In particular, Rees et al have proposed that extragalactic radio sources that are not luminous in the optical are explainable by massive black holes with a low accretion rate. ${ }^{27}$ In particular, the model can be applied to Sgr A*. ${ }^{28}$

\section{UPPER LIMITS TO THE MASS OF SGR A* ?}

With the advent of near-IR imaging arrays, astrometry of near-IR sources has become much more reliable, and the positional offset between Sgr A* and the components of IRS16 has been established reliably. ${ }^{29}$ Arguments to place an upper limit on the mass of Sgr A* have been made based on this positional offset, ${ }^{30,31}$ since it has always been assumed that IRS16 is the core of the central star cluster and marks the dynamic center of the Galaxy. However, evidence is mounting that the components of IRS16 are best explained as young stars. ${ }^{32}$ Until the location of the dynamic center is determined independently, such arguments are not definitive.

Furthermore, the broad $\mathrm{He}$ and $\mathrm{H}$ lines assumed to arise from the wind of a single central source ${ }^{30}$ may be simply stellar winds of Wolf-Rayet stars. ${ }^{34}$ This means that upper limits to the mass of the central source based on the Eddington 1imit may not be appropriate. ${ }^{31}$ To account for the compact $0.51 \mathrm{Mev} \gamma$-ray $\mathrm{e}^{+} \mathrm{e}^{-}$annihilation line from the Galactic center, one may conclude that the positrons have to be produced via photon-photon pair production in a very compact volume, thereby setting an upper limit to the mass of the possible black-hole. ${ }^{35}$ Since the identification of the $\gamma$-ray line with the Galactic center is highly uncertain and there are alternate ways to produce positrons,,$^{36}$ the arguments are not definitive.

The non-association of a luminous source with Sgr A* does not preclude the possibility of a massive black-hole, since in the case of low accretion rate onto a massive black-hole, which could explain Sgr A*, little luminosity is expected from the ion-supported accretion disk. In addition, the actual accretion process is highly uncertain, Sgr A* may be in a "starved" phase. 27 


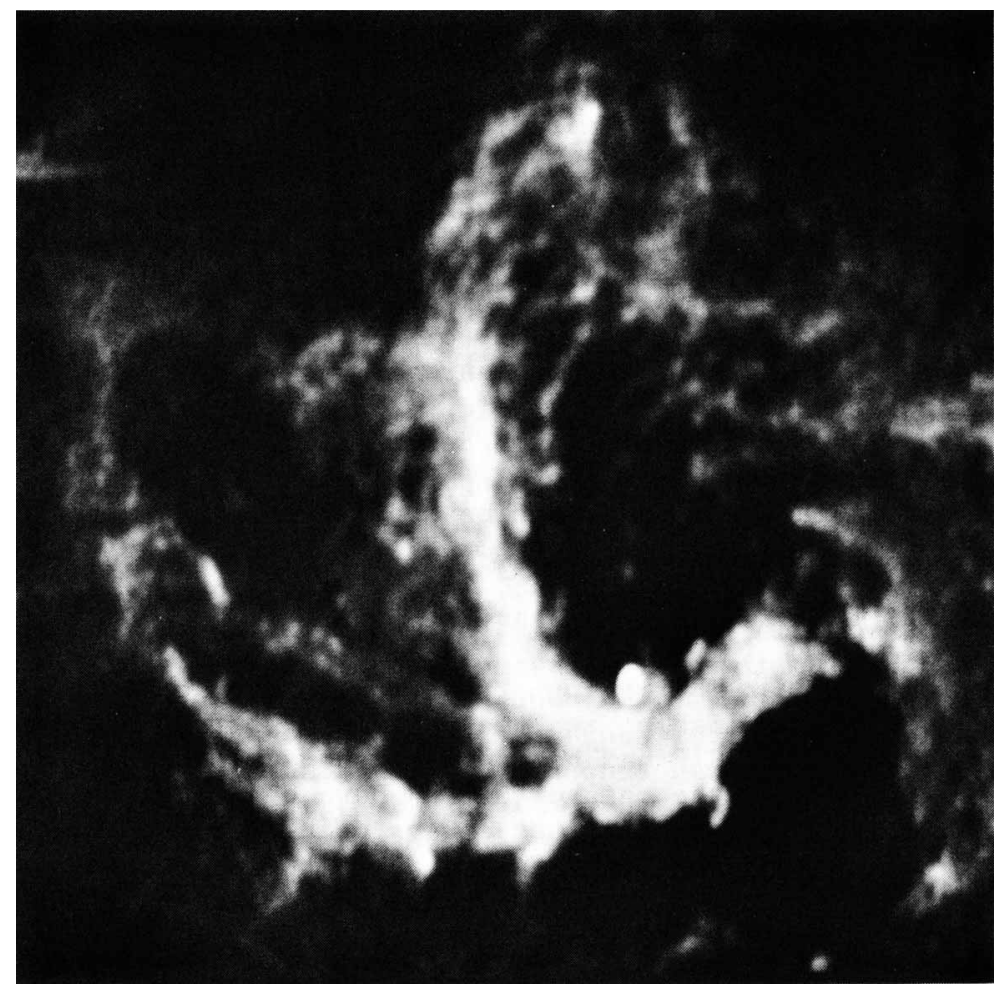

Figure 1: $\lambda 6 \mathrm{~cm}$ VLA A-configuration radio image of the ionized gas within the inner $2.2 \mathrm{pc}$ of the Galaxy at a resolution of $0.6 " \mathrm{x}$ $0.3 "$. 
The most direct observational indication of a concentrated mass at the center is still given by the [NeII] measurements of the large velocities of the ionized gas. ${ }^{37}$ Given the complexities of the ionized gas distribution (figure 1 ) and the uncertain origin of its motion, the gas velocities may not probe the gravitational potential. The origin and motion of the ionized gas will become clearer if the three-dimensional velocity field of the gas is known. Masson et al (this Conferenice) have attempted to measure the proper motion of the ionized gas by comparing two high-resolution VLA maps, obtained 4.5 years apart. A transverse motion of $100 \mathrm{~km} \mathrm{~s}^{-1}$ corresponds to a proper motion of 0.01 " in this period. This proper motion measurement can only improve with time as the time base increases.

\section{CONCLUSION}

Accretion onto massive black-holes was proposed to account for the inordinately large luminosity observed from a very compact volume in quasars and radio galaxies. There is almost no hope of ever spatially resolving these collapsed objects at such great distances. In the center of our Galaxy, the proximity permits probing within scales approaching the Schwarzschild radius of a $10^{6} \mathrm{M} /$ black-hole, as in the case of $\mathrm{Sgr}$ $A^{*}$. Ironically, while the unique properties of $\mathrm{Sgr} \mathrm{A}^{*}$ preclude models involving known stellar systems, the radio luminosity is too low to demand the mechanism of accretion onto a massive black hole. The exact nature of Sgr A* can only be resolved with more observations. The best instrument to do so will be the Very Long Baseline Array (VLBA).

\section{REFERENCES}

1. Balick, B., and Brown, R. L., 1974, Ap. J., 194, 265.

2. Lo, K. Y. et al, 1985, Nature, 315, 124.

3. Lynden-Be11, D., and Rees, M. J., 1971, MNRAS, 152, 461.

4. Ekers, R. D., Lynden-Be11, D., 1971, Ap. Lett., 9, 189.

5. Downes, D., Martin, A., 1971, Nature, 233, 112.

6. Lo, K. Y., 1987, AIP Proc. 155: Symposium honoring C. H. Townes, ed. D. C. Backer, (AIP; New York), 155, 30.

7. Kellerman, K. I., Shaffer, D. B., Clark, B. G., and Geldzahler, Clark B. G. , 1977, Ap. J. (Lett.), 214, L61.

8. Lo, K. Y., Cohen, M. H., Readhead, A. C. S., and Backer, D. C., 1981, Ap. J., $249,504$.

9. Davies, R. D., Walsh, D., and Booth, R., 1976, MNRAS, 177, 319.

10. Marcaide, J. M., et al, 1985, Conference on Active Galactic Nuclei, (ed. Dyson, J. E.; Manchester University Press), p.53.

11. Zhou, J.H., Ekers, R., Goss, M., Lo, K. Y., Narayan, R., this volume.

12. Zy1ka, R., Mezger, P. G., 1988, Ast. \& Ap., 190, L25.

13. Ozernoi, L. M., Shishov, V. I., 1977, Sov. Astron. (Lett.), 3, 233.

14. Rickett, B., 1986, Ap. J., 307, 564.

15. Rickett, B., Coles, W., Bourgois, G., 1984, Ast. \& Ap., 134, 390. 
16. Romani, R., Narayan, R., Blandford, R., 1986, MNRAS, 220, 19.

17. Backer, D. C., Sramek, R., 1982, Ap. J., 260, 512.

18. Backer, D.C., Sramek, R., 1987, AIP Proc. 155: Symposium honoring C.H. Townes, ed. D. C. Backer, (AIP; New York), 155, 163.

19. Cordes, J., Ananthakrishnan, S., and Dennison, B., 1984, Nature, 309, 689.

20. Reynolds, S., McKee, C., 1980, Ap. J., $239,893$.

21. Engelke, C. , 1988, Ap. J., 325, 153.

22. Gusten, R., et al., 1987, Ap. J., 318, 124.

23. Barte1, N. et a1, 1982, Ap. J., 262, 556.

24. Bash, F., Kaufman, M., 1986, Ap. J., 310, 621.

25. Kellermann, K., Pauliny-Toth, I., 1981, Ann. Rev. Ast. \& Ap., 19, 373.

26. Begelman, M., Blandford, R., Rees, M., 1984, Rev, for Mod. Phys,, 56, 255.

27. Rees, M., Begelman, M., Blandford, R., Phinney, E., 1982, ature, $295,17$.

28. Rees, M., 1987, AIP Proc. 155: Symposium honoring C. H. Townes, ed. D.C.Backer, (AIP; New York), 155, 71.

29. Forrest, W., Shure, M., Pipher, J., Woodward, C., 1987, AIP Proc. 155: Symposium honoring C. H. Townes, ed. D. C. Backer, (AIP; New York), 155, 153.

30. Gurzadyan, V. G., Ozernoi, L. M., 1980, Ast. \& Ap, 86, 315.

31. Ozernoi, L. M., 1987, AIP Proc. 155: Symposium honoring C. H. Townes, ed. D. C. Backer, (AIP; New York), 155, 181.

32. Allen, D. A., 1987, AIP Proc. 155: Symposium honoring C. H. Townes, ed. D. C. Backer, (AIP; New York), 155, 1.

33. Hall, D. N. B., Kleinman, S. G., and Scoville, N. Z., 1982, Ap.J. (Lett.), 262, L53.

34. Allen, D. A., this volume.

35. Ramaty, R., and Lingenfelter, R. E., 1983, Highlights of Astronomy, 6,525 .

36. Blandford, R. D., 1982, AIP Conf. Proc. 83, ed. G. Riegler, R. Blandford, (AIP; New York), 177.

37. Serabyn, G., Lacy, J., 1985, Ap. J., 293, 445. 